\title{
Platelets, alcohol consumption, and onset of brain infarction
}

\author{
Heikki Numminen, Matti Hillbom, Seppo Juvela
}

\begin{abstract}
Objectives-Previous investigations have suggested that recurrent rebound thrombocytosis after alcohol misuse may be a factor in the pathogenesis of thromboembolic disease. Alcohol consumption, platelet count, and platelet function were examined among patients of working age with brain infarction.

Methods-Platelet count and risk factors for stroke were studied in 426 stroke patients and 157 control patients in hospital. The measures were platelet count obtained within four days after the stroke onset, in vitro adenosine diphosphate induced platelet aggregation, associated thromboxane $B 2$ formation, and urinary excretion of 11-dehydrothromboxane B2. Results-After adjustment for sex, age, cardiac disease, diabetes, and alcohol intake, hypertension (OR 3.4, 95\% confidence interval $(95 \%$ CI) $2 \cdot 0-6 \cdot 0)$ and current smoking (OR $2 \cdot 1,95 \%$ CI $1 \cdot 4-3 \cdot 3$ ) were associated with an increased risk for brain infarction. Platelet count shortly after the onset of disease was higher in the stroke patients than in the controls (OR $1 \cdot 05 / 10^{10} / 1$ platelets; $95 \%$ CI $\left.1 \cdot 02-1 \cdot 09\right)$. The patients with brain infarction who were heavy alcohol drinkers $(n=144)$ showed both thrombocytosis (OR 2.30, 95\% CI $0 \cdot 82-6 \cdot 44)$ and thrombocytopenia (OR $3 \cdot 20,95 \%$ CI $1 \cdot 19$ to $8 \cdot 59$ ) more often at the onset of the stroke than the other patients with brain infarction. The thromboxane variables showed inconsistent associations with the onset of stroke. There was no consistent platelet abnormality among alcohol misusers at the onset of ischaemic brain infarction.

Conclusions-Alcohol induced thrombocytopenia and rebound thrombocytosis were both often seen at the onset of brain infarction in patients who were heavy alcohol drinkers. Therefore, other mechanisms which could contribute to the high frequency of recurrences of ischaemic stroke among heavy drinkers should be investigated.
\end{abstract}

$(\Im$ Neurol Neurosurg Psychiatry 1996;61:376-380)

Keywords: cerebral infarction; alcohol induced rebound thrombocytosis; platelet thromboxane formation

The role of platelets in the pathogenesis of vascular diseases is important. Platelet count and function after the onset of acute stroke and among patients with previous stroke have been intensively studied, but few studies deal with reactive thrombocytoses, although such conditions can be considered to contribute to stroke onset as predisposing factors. Probably the most common of the reactive thrombocytoses is rebound thrombocytosis after alcohol misuse, a condition which, in about one third of the patients, follows acute withdrawal from prolonged heavy alcohol drinking. ${ }^{1}$ Haselager and Vreeken described, in 1977, two alcoholic men having reactive thrombocytosis concomitant with an unexplained recurrent venous thrombosis and pulmonary embolism and they hypothesised that the alcohol induced rebound thrombocytosis could precipitate thromboembolic disease. ${ }^{2}$ Heavy drinking of alcohol has been found to be a risk factor for ischaemic stroke ${ }^{34}$ and also the rate of recurrences of ischaemic stroke seems to increase in patients who are heavy drinkers. ${ }^{5}$

As we have also seen alcoholic patients with recurrent thromboembolic diseases and thrombocytosis, we became interested in the role of platelets and alcohol consumption at the onset of brain infarction. The transient rebound thrombocytosis induced by alcohol and the concurrent enhancement of platelet function could be considered to provoke thrombus formation. Assuming that rebound thrombocytosis could act as a predisposing factor for ischaemic stroke, we examined whether increased platelet count and function are frequent findings at the onset of ischaemic stroke in patients of working age who are current heavy alcohol drinkers.

\section{Patients and methods}

PATIENTS

We examined 426 consecutive stroke patients ( 110 women and 316 men) and 157 control patients ( 75 women and 82 men) of working age who were admitted as emergencies to the Helsinki University Central Hospital. All these patients, aged from 16 to 59 years, had data on alcohol consumption and platelet count. The stroke patients had had their first ever ischaemic brain infarction and they included 144 heavy alcohol drinkers. They were compared both with the control patients and those stroke patients who were not heavy drinkers. All the patients were admitted to hospital within 24 hours after the onset of the first symptoms of the disease. The hospital receives patients from a defined region and most of those stricken by stroke in these age groups and living in the region are immediately 
referred to the hospital. The controls were patients who were taken to the same hospital on an emergency basis because of acute appendicitis $(\mathbf{n}=91)$, acute dyspnoea ( $\mathbf{n}=$ 23), cholecystitis $(n=19)$, nephrolithiasis ( $=10)$, and other acute diseases $(n=14)$. These medical emergencies were not considered to accumulate among either heavy drinkers or teetotallers. Unfortunately, the control patients were few, but this was due to the fact that they were also consecutive patients from the same time period. The study protocol was approved by the ethics committee of the Helsinki University Central Hospital.

All the patients and controls were interviewed by a trained interviewer within 48 hours of admission according to a structured questionnaire. If they were unable to give an interview, the relatives and friends were interviewed. The questionnaire included questions on the height and weight of the patient; previous diseases; recent drug use including analgesics; recent drinking of coffee, beer, wine, and spirits; and current and previous smoking habit.

The occurrence of previously diagnosed hypertension, diabetes, and hyperlipaemia were recorded. The patients were considered to have definite hypertension if their blood pressure readings before the index admission had repeatedly exceeded $160 \mathrm{~mm} \mathrm{Hg}$ systolic pressure or $95 \mathrm{~mm} \mathrm{Hg}$ diastolic pressure before the illness or if they used antihypertensive medication. Cardiac disease included current atrial fibrillation, previously diagnosed myocardial infarction, and congestive heart failure. The body mass index (BMI, calculated as weight $/$ height $^{2}$ in $\mathrm{kg} / \mathrm{m}^{2}$ ) was used as the index of relative weight.

Recent alcohol consumption was recorded as $g$ ethanol consumed within one week before the onset of the brain infarction. A standard drink in Finland contains $12 \mathrm{~g}$ ethanol. Problem drinking was assessed with the short CAGE questionnaire, which has been described in detail elsewhere. ${ }^{6}$ The CAGE interview includes four questions on abnormal drinking behaviour, and patients with two or more positive answers are considered CAGE positive; CAGE positivity is a sensitive indicator of previous and current extensive drinking. The patients who were CAGE positive or whose regular weekly alcohol intake exceeded $300 \mathrm{~g}$ ethanol were included in the category of heavy drinkers.

\section{LABORATORY PROCEDURES}

Blood samples for measurements of platelet count were taken from all the patients within three days of admission. Further samples were taken from a random subsample of 13 patients one week and more than two weeks after the onset of stroke. None of these 13 patients had taken any drugs known to influence platelet function during the week before admission.

The studies of platelet function included adenosine diphosphate induced irreversible platelet aggregation and associated thromboxane B2 (TxB2) formation. Blood was collected with minimal stasis via a plastic cannula into tubes containing $3 \cdot 8 \%$ sodium citrate. Platelet rich plasma (PRP) and platelet poor plasma (PPP) were centrifuged and used to adjust the platelet count of PRP to $250 \times 10^{9} / 1$. Platelet aggregations were measured with a dual channel aggregometer (Chrono-Log, Coulter Electronics Ltd, UK) starting 45 minutes after the blood sampling. Aggregation in PRP was induced by adenosine diphosphate (final concentration 8 or $16 \mu \mathrm{M}$ ) to yield an irreversible aggregation. The reaction was stopped by 1 $\mathrm{mmol} / 1 \mathrm{HCl}$ after five minutes and aggregated PRP samples were frozen for further analyses. Thromboxane B2 was measured from aggregated PRP by a radioimmunoassay (Amersham International, UK). In addition, 24 hour urine samples were collected from a random subsample of 11 patients for urinary 11-dehydrothromboxane B2 analyses. This is a stable metabolite of TxA2 in urine, which could reflect platelet aggregation in vivo. ${ }^{78} 11$ DehydroTxB2 was analysed by enzyme linked immunosorbent assay (ELISA) (Cayman Co, USA) from samples taken on the same days as the blood samples for platelet function measurements.

The erythrocyte mean volume (MCV, normal range, 80 to $96 \mathrm{fl}$ ) was used as a laboratory marker of alcohol consumption, because it was routinely available for most patients $(92 \%)$ and controls (99\%). Besides heavy drinking, cigarette smoking has also been shown to raise $\mathrm{MCV}^{9}$ and diabetic patients may have slightly raised $\gamma$ glutamyl transferase (GGT). The value (normal range, 0-45 U/l) was available for 393 patients (92\%) and 94 $(60 \%)$ control patients.

\section{STATISTICAL METHODS}

The data were analysed with the BioMedical Data Package statistical programs (BMDP Statistical Software Inc, version 1993, University of California at Los Angeles). The categorical variables were compared with the Pearson $\chi^{2}$ test, the test for linear trend, or the Mantel-Haenszel statistics. The continuous variables, which were expressed as mean (SD) or median with the 25 th and 75 th percentiles (if the distribution was skewed), were compared with the Student's $t$ test, Mann-Whitney $U$ test, or Spearman's rank correlation coefficients $(r s)$ when appropriate. The odds ratios (OR) with $95 \%$ confidence intervals (95\% CIs) before and after adjustment for potential confounding variables were calculated by logistic regression. The $95 \%$ CIs for the logistic coefficients were estimated using the standard errors. In all statistical testing performed the significance was accepted at $\mathrm{P}<0.05$.

\section{Results}

Table 1 presents the baseline characteristics, previous diseases, and health habits of the patients. The occurrence of most cardiovascular risk factors was more frequent in the patients than the control patients. Only hyperlipaemia and atrial fibrillation showed a nonsignificant difference. The GGT values were 
Table 1 Baseline characteristics, previous diseases, and health habits in patients with brain infarction and control patients

\begin{tabular}{lcc}
\hline & Patients & Controls \\
\hline Patients (n) & 426 & 157 \\
Age (mean (SD), y) & $47 \cdot 5(9 \cdot 7)^{\star}$ & $43 \cdot 2(12 \cdot 4)$ \\
BMI (mean (SD), kg/m $\left.{ }^{2}\right)$ & $26 \cdot 2(4 \cdot 6)^{\star}$ & $25 \cdot 2(4 \cdot 4)$ \\
Platelet count (mean (SD), $\left.\times 10^{9} / 1\right)$ & $244(87)^{\star}$ & $227(67)$ \\
Hypertension (n (\%)) & $182(43)^{\star}$ & $24(15)$ \\
Current smoking (n (\%)) & $235(55)^{\star}$ & $56(36)$ \\
Heavy drinking (n (\%)) & $144(34)^{\star}$ & $30(19)$ \\
Recent alcohol consumption & & \\
(g/week (n (\%))): & $136(32)$ & $67(43)$ \\
0 & $160(38)$ & $74(47)$ \\
1-150 & $62(14)$ & $11(7)$ \\
151-300 & $68(16)^{\star}$ & $5(3)$ \\
$>300$ & $113(27)^{\star}$ & $24(15)$ \\
Cardiac disease (n (\%)) & $47(11)^{\star}$ & $6(4)$ \\
Myocardial infarction (n (\%)) & $49(12)^{\star}$ & $9(6)$ \\
Diabetes mellitus (n (\%)) & $57(13)$ & $14(9)$ \\
Hyperlipaemia (n (\%)) & $27(6)$ & $8(5)$ \\
Atrial fibrillation (n (\%)) & 393 & 94 \\
GGT (U/l): & $147(37)^{\star}$ & $15(16)$ \\
Samples (n) & 424 & 156 \\
MC (n (\%)) & $57(13)$ & $16(10)$ \\
Samples (n) & & \\
Sa 97 (\%) & & \\
\hline
\end{tabular}

${ }^{\star} \mathrm{P}<0.05 v$ control subjects.
$\mathrm{GGT}=\gamma$-Glutamyl transferase; $\mathrm{MVC}=$ erythrocyte mean volume; $\mathrm{BMI}=$ body mass index.

Table 2 Multivariate odds ratios (ORs) of brain infarction

\begin{tabular}{lll}
\hline & $O R$ & $95 \% C I$ \\
\hline Hypertension & 3.43 & $1 \cdot 98-5 \cdot 95$ \\
Current smoking & $2 \cdot 12$ & $1 \cdot 35-3 \cdot 33$ \\
Platelet count (per $\left.1 \times 10^{1 \%} / 1\right)$ & 1.05 & $1 \cdot 02-1 \cdot 09$ \\
Recent alcohol consumption & & \\
(g/week $(\%))$ : & & \\
$1-150$ & 0.98 & $0 \cdot 62-1 \cdot 55$ \\
$151-300$ & 1.76 & $0 \cdot 79-3.97$ \\
$>300$ & 4.92 & $1.64-14 \cdot 72$ \\
\hline
\end{tabular}

The analysis includes data on 398 patients with brain infarction and 154 control patients. The ORs represent comparisons with patients without a risk factor. The ORs have been adjusted for the listed risk factors and for age, sex, cardiac disease, myocardial infarction, diabetes, hyperlipaemia, atrial fibrillation, and heavy drinking.

more than the normal range in $147(37 \%)$ of the patients and in $15(16 \%)$ of the control patients. However, the prevalence of raised MCV values did not significantly differ between the groups. Thirty per cent of the patients had drunk more than $150 \mathrm{~g}$ ethanol during the week preceding the brain infarction, whereas the corresponding figure for the

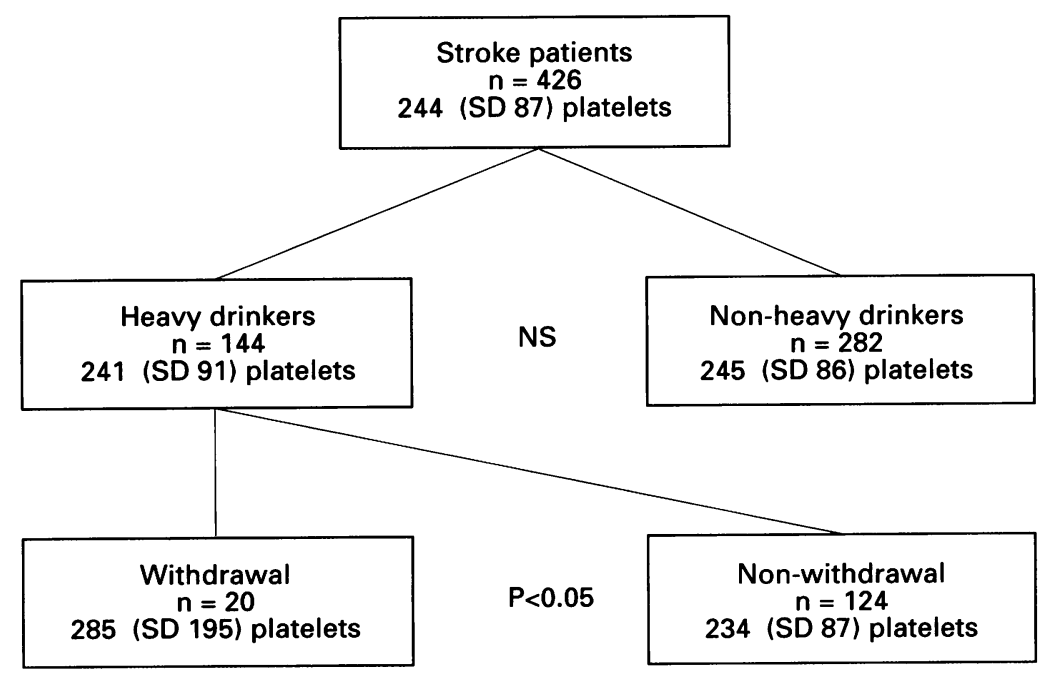

Similarities and differences in platelet counts between the various groups of stroke patients. control patients was $10 \%$. Thus both the GGT and the alcohol drinking data suggested that heavy drinkers were more frequent among the patients with brain infarction than the controls.

Platelet count on admission was significantly higher among the patients with brain infarction than the control patients. High platelet count remained an independent risk factor in the multivariate analysis after adjustment of the odds ratios for other risk factors (table 2), even for recent alcohol consumption and a history of heavy drinking.

Among the patients with brain infarction who were heavy drinkers $(144,34 \%)$, we found higher than normal platelet counts $\left(>300 \times 10^{9} / 1\right)$ on admission in $18 \%$. Among the other patients with brain infarction (282, $66 \%$ ), the corresponding figure was $15 \%$. Lower than normal platelet counts $(<151 \times 10 \%$ ) were found in $10 \%$ and $4 \%$ respectively. The risk of heavy drinkers having a higher or lower than normal platelet count on admission was non-significant, if alcohol consumption during the preceding week before brain infarction was not taken into account (figure).

However, if the heavily drinking patients with brain infarction had abstained from alcohol drinking during the week preceding the onset of stroke they had more frequent platelet counts over $300 \times 10^{9} / 1$ than the patients with brain infarction who were not heavy drinkers (OR 2.30, 95\% CI 0.82-6.44), but if they had been drinking alcohol regardless of the amount, they more often had platelet counts under $151 \times 10^{9} / 1 \quad$ (OR 3.20, 95\% CI 1.19-8.59). Accordingly, the heavily drinking patients with brain infarction showed both high and low platelet counts more often than the other patients with brain infarction. There were eight patients with brain infarction with thrombocytosis $\left(>450 \times 10^{9} / 1\right)$ and three of them had a probable rebound thrombocytosis caused by withdrawal from recent heavy drinking of alcohol.

Formation of TxB2 during adenosine diphosphate induced platelet aggregation in vitro was serially analysed from plasma samples of 13 patients with brain infarction. All the patients had normal platelet counts on admission. Formation of TxB2 varied depending on how much alcohol the patients had consumed during the preceding week. In eight patients, two of whom were non-drinkers and six who had consumed less than $300 \mathrm{~g}$ ethanol during the preceding week of the index stroke (one was a heavy drinker who had been abstinent for several weeks), TxB2 formation decreased (the median decrease between the value on admission and the value one week later was $-70 \mathrm{fmol} / 10^{7}$ platelets, 25 th and 75 th percentiles -359 and +67 ), whereas in five patients who had consumed over $300 \mathrm{~g}$ ethanol, TxB2 formation increased (the median increase was $+688,25$ th and 75 th percentiles +136 and +1157 ). The difference between the changes was significant $(P<0.05)$. Similar changes were found when $\mathrm{TxB} 2$ formation was calculated as the product 
of $\mathrm{TxB} 2$ formed per $10^{7}$ platelets and the platelet count in whole blood.

Urinary 11-dehydroTxB2 values were available for 11 patients. The range varied from 261 to $3552 \mathrm{pmol} / \mathrm{mmol} / \mathrm{g}$ urinary creatinine. The values did not show any significant correlations with the corresponding platelet counts, aggregation percentages, or values for formation of $\mathrm{TxB} 2$ in vitro.

\section{Discussion}

Platelet count was shown to associate positively with risk of ischaemic stroke after adjustment for potential risk factors, including habitual heavy drinking and recent alcohol consumption. Higher than normal platelet counts on admission were more frequent among the patients with brain infarction than among the control patients. The multivariate analysis indicated that a high platelet count was independently associated with the onset of brain infarction.

There is no uniform agreement whether platelet count is increased or decreased at onset of stroke. Platelet count may be decreased because platelets are consumed during thrombogenesis. ${ }^{1011}$ However, not all investigations agree with this notion, but rather suggest that thrombocytopenia and associated large platelets are likely to precede the vascular event. ${ }^{12}$ Higher than normal platelet counts have also been reported to occur in patients with brain infarction. ${ }^{13}$ The above mentioned findings were made among elderly stroke patients and our study cannot consider this question, because we did not know the platelet count immediately before the onset of stroke in our patients. At best, we can say that there is a relation between the occurrence of brain infarction and raised platelet counts immediately after the onset of stroke. However, it is difficult to believe that brain infarction had, within three days, caused the pronounced thrombocytosis which occurred in eight patients.

The factors known to provoke reactive thrombocytosis include exercise, haemorrhage, acute and chronic inflammatory disorders, malignant diseases, haemolysis, splenectomy, major surgery, certain drug treatments, and withdrawal from prolonged alcohol drinking. Infections and heavy drinking are of particular interest, as they often precede the onset of ischaemic stroke and have been shown to be independent risk factors for brain infarction. ${ }^{341415}$

Our main purpose was to show whether rebound thrombocytosis coincides with the time of onset of stroke among heavy drinkers of alcohol. Bias could have been possible through missing the heavy drinkers who had stopped drinking alcohol earlier than one week before the onset of the index disease. This could have led to an increased frequency of high platelet counts among the patients who were not classified as heavy drinkers. However, by using the positive answers to the CAGE questions we were able to include such patients into the group of heavy drinkers.
Thus bias due to hidden alcohol related rebound thrombocytosis is improbable. It is also unlikely that the acute diseases of the control patients could have caused haemodilution or platelet consumption and thereby decreased the platelet count. Both low and high platelet counts were found in heavily drinking patients on admission for brain infarction. Altogether, eight patients with brain infarction had definite thrombocytosis $\left(>450 \times 10^{9} / 1\right)$. Three of them had thrombocytosis because of withdrawal from recent prolonged heavy drinking of alcohol. Accordingly, alcohol related rebound thrombocytosis was not a very common finding among the patients with brain infarction who were recent heavy drinkers. We also found 15 recent heavy drinkers who had thrombocytopenia $(<151 \times 109 / 1)$.

In fact, we cannot exclude the possibility that the alcohol induced rebound thrombocytosis contributed to the onset of ischaemic stroke in three of our patients. However, as recent heavy drinkers rather often had normal platelet counts and even obvious thrombocytopenia at the onset of stroke and showed an increase in platelet count and function within a week after admission-that is, several days after the onset of ischaemic stroke, the increased risk for ischaemic stroke and recurrence of stroke among heavy drinkers should be considered to result from other factors, such as cardiac insufficiency due to alcoholic cardiomyopathy and arrhythmias.

Enhanced platelet aggregability often associates with the onset of ischaemic stroke, ${ }^{16-21}$ but it may also exist after onset of haemorrhagic stroke. ${ }^{22}$ Platelet hyperfunction may precede the onset of stroke ${ }^{23}$ or develop after onset. ${ }^{24}$ In the presence of essential and reactive thrombocytoses platelet hyperaggregability may precipitate the onset of stroke. ${ }^{23}{ }^{25-27} \mathrm{It}$ has been reported that three patients developed brain infarction in association with reactive thrombocytosis in the late recovery period (10-25 days) after cardiopulmonary bypass surgery $^{28}$ and that an alcoholic patient developed a transient ischaemic attack while having transient thrombocytosis and enhanced platelet aggregability due to cessation of prolonged heavy drinking. ${ }^{29}$ The few measurements of platelet function in our study were not enough to prove whether enhanced platelet function played a relevant part in the onset of stroke in our patients.

In patients having thrombocytopenia due to ethanol toxicity, the platelets are larger than normal. ${ }^{30}$ On the other hand, large platelets are more reactive than small platelets. ${ }^{31}$ Therefore, it is also possible that increased platelet reactivity already occurs in the presence of a low platelet count shortly after the cessation of drinking among recent heavy drinkers of alcohol, although platelet reactivity is usually decreased during prolonged heavy alcohol consumption. ${ }^{32}$

In conclusion, the contribution of reactive thrombocytoses to the onset of ischaemic brain infarction remains unclear. Rebound thrombocytosis due to cessation of prolonged heavy alcohol drinking was found to be an 
infrequent finding at the onset of acute ischaemic brain infarction. Recent heavy drinkers often showed lower than normal platelet counts. Therefore, other mechanisms which could contribute to the high frequency of ischaemic stroke recurrences among heavy drinkers should be investigated.

This study was supported in part by grants from the Yrjö Jahnsson Foundation, the Oulu Medical Foundation, and the Neurology Foundation, Finland. We thank Mrs Saija Eirola for her skillful technical assistance.

1 Sullivan LW, Herbert V. Suppression of hematopoiesis by ethanol. 7 Clin Invest 1964;43:2048-62.

2 Haselager EM, Vreeken J. Rebound thrombocytosis after alcohol abuse: a possible risk factor in the pathogenesis of thromboembolic disease. Lancet 1977;i:774-5.

3 Gill JS, Zezulka AV, Shipley MJ, Gill SK, Beevers DG. Stroke and alcohol consumption. $N$ Engl $\mathcal{F}$ Med 1986; 315:1041-6.

4 Hillbom $M$, Haapaniemi $H$, Juvela $S$, Palomõki $H$, Numminen $H$, Kaste $M$. Recent alcohol consumption, cigarette smoking, and cerebral infarction in young adults. Stroke 1995;26:40-5

5 Sacco RL, Shi T, Zamanillo MC, Kargman DE. Predictor of mortality and recurrence after hospitalized cerebra infarction in an urban community: the northern Manhattan stroke study. Neurology 1994;44:626-34.

6 Bush B, Shaw S, Cleary P, Delbanco TL, Aronson MD Screening for alcohol abuse using CAGE questionnaire. Am ₹ Med 1987;82:231-5.

7 Catella F, Healy D, Lawson JA, FitzGerald GA. 11 Dehydrothromboxane B2: a quantitative index of thromboxane A2 formation in the human circulation. Proc Natl Acad Sci 1986;83:5861-5.

8 Ciabattoni G, Pugliese F, Davi G, Pierucci A, Simonett BM, Patrono C. Fractional conversion of thromboxane B2 to urinary 11-dehydro-thromboxane B2 in man. Biochem Biophys Acta 1989;992:66-70.

9 Papoz L, Warnet J-M, Pequignot G, Eschwege E, Claude $\mathrm{JL}$, Schwartz D. Alcohol consumption in a healthy population: relationship to gamma-glutamyl transferase activity and mean corpuscular volume. $\mathscr{F} A M A 1981$ 345: 1748-51.

10 D'Erasmo E, Aliberti G, Celi FS, Romagnoli E, Vecci E, Mazzuoli GF. Platelet count, mean platelet volume and their relation to prognosis in cerebral infarction. $f$ Int Med 1990;227:11-4.

11 Tohgi H, Suzuki H, Tamura K, Kimura B. Platelet volume, aggregation and adenosine triphosphate release in ume, aggregation and adenosine triphosph

12 O'Malley T, Langhorne P, Elton RA, Stewart C. Platelet .

13 Valkila EH, Salenius JP, Koivula TA. Platelet indices in patients with occlusive carotid artery disease. Angiology 1994;45:361-5.

14 Syrjönen J, Valtonen VV, Iivanainen $M$, Kaste $M$ Huttunen JK. Preceding infection as an important risk factor for ischaemic brain infarction in young and middle aged patients. $B M \mathcal{F} 1988 ; 296: 1156-60$.
15 Grau AJ, Buggle F, Heindl S, Steichen-Wiehn C, Banerjee $\mathrm{T}$, Maiwald $\mathrm{M}$, et al. Recent infection as a risk factor for cerebrovascular ischemia. Stroke 1995;26:373-9.

16 Dougherty JH, Lewy DE, Weksler BB. Platelet activation in acute cerebral ischaemia. Serial measurements of platelet function in cerebrovascular disease. Lancet 1977; platelet finc $821-4$.

17 Mulley GP, Heptinstall S, Taylor PM, Mitchell JRA. ADPinduced platelet release reaction in acute stroke. Thromb induced platelet release

18 Koudstaal PJ, Ciabattoni G, van Gijn J, Nieuwenhuis HK, de Groot PG, Sixma JJ, Patrono C. Increased thromboxane biosynthesis in patients with acute cerebral ischemia. Stroke 1993;24:219-23.

19 Uchiyama S, Yamazaki M, Maruyama S, Handa M, Ikeda $\mathrm{Y}$, Fukuyama $\mathrm{M}$, et al. Shear-induced platelet aggregation in cerebral ischemia. Stroke 1994;25:1547-51.

20 van Kooten F, Ciabattoni G, Patrono C, Schmitz PIM, van Gijn J, Koudstaal PJ. Evidence of episodic platelet activation in acute ischemic stroke. Stroke 1994;25:278-81.

21 D'Andrea G, Cananzi AR, Perini F, Hasselmark L, Alecci M, Fortunato A, Welch KMA. Platelet function in acute ischemic stroke: relevance of granule secretion. Cerebrovasc Dis 1994;4:163-9.

22 Liu L, Lin Z, Shen Z, Zhang G, Li S, Cao P. Platelet hyperfunction exists in both acute non-haemorrhagic and hyperfunction exists in both acute non-haemorrhagic

23 Preston FE, Martin JF, Stewart RM, Davies-Jones GAB Thrombocytosis, circulating platelet aggregates, and neurological dysfunction. $B M F$ 1979;2:1561-3.

24 Uchiyama S, Takeuchi M, Osawa M, Kobayashi I, Maruyama S, Aosaki M, Hirosawa $K$. Platelet function tests in thrombotic cerebrovascular disorders. Stroke 1983;14:511-7.

25 Casto L, Camerlingo M, Finazzi G, Censori B, Barbui T, Mamoli A. Essential thrombocythaemia and ischemic stroke: report of six cases. Ital $\mathcal{F}$ Neurol Sci 1994;15: 359-62.

26 Arboix A, Besses C, Pilar A, Massons JB, Florensa L Oliveres $M$, Sans-Sabrafen J. Ischemic stroke as first manifestation of essential thrombocythemia. Report of manifestation of essential throm

27 Heptinstall S, Cockbill SR, Burgess-Wilson ME, Jones $\mathrm{EW}$. Studies on platelets before and after stroke in a patient with essential thrombocythaemia. $B M F$ 1988;296: 1298-9.

28 Crowley JJ, Hannigan M, Daly K. Reactive thrombocytosis and stroke following cardiopulmonary bypass surgery: case repor

29 Neiman J. Association of transient ischemic attack in alcohol withdrawal with changes in haemostasis. British fournal of Addiction 1988;83:1457-9.

30 Levin J, Bessman JD. The inverse relation between platelet volume and platele number. Abnormalities in hematologic disease and evidence that platelet size does not corlogic disease and evidence that platelet size does not correlate with

31 Thompson CB, Eaton KA, Princiotta SM, Rushin CA, Valeri CR. Size dependent platelet subpopulation relationships of platelet volume to ultrastructure enzymatic activity and function. $\mathrm{Br} \mathcal{F}$ Haematol 1982;50:509-19.

32 Mikhailidis DP, Jenkins WJ, Barradas MA, Jeremy JY, Dandona P. Platelet function defects in chronic alcoholism. BMf 1986;293:715-8. 